\title{
Ab initio prediction of magnetically dead layers in freestanding $\gamma$-Ce(111)
}

\author{
S. Jalali Asadabadi ${ }^{1, *}$ and F. Kheradmand ${ }^{1}$ \\ ${ }^{1}$ Department of Physics, Faculty of Science, University of Isfahan (UI), Hezar Gerib Avenue, Isfahan 81744, Iran
}

(Dated: July 24, 2018)

\begin{abstract}
It is well known that the surface of nonmagnetic $\alpha$-Ce is magnetically ordered, i.e., $\gamma$-like. One then might conjecture, in agreement with previous theoretical predictions, that the $\gamma$-Ce may also exhibit at its surfaces even more strongly enhanced $\gamma$-like magnetic ordering. Nonetheless, our result shows that the (111)-surfaces of magnetic $\gamma$-Ce are neither spin nor orbitally polarized, i.e., $\alpha$-like. Therefore, we predict, in contrast to the nonmagnetic $\alpha$-phase which tends to produce magnetically ordered $\gamma$-like thin layers at its free surfaces, the magnetic $\gamma$-phase has a tendency to form $\alpha$-like dead layers. This study, which explains the suppressed (promoted) surface magnetic moments of $\gamma$-Ce $(\alpha$-Ce), shows that how nanoscale can reverse physical properties by going from bulk to the surface in isostructural $\alpha$ - and $\gamma$-phases of cerium. We predict using our freestanding surface results that a typical unreactive and non-diffusive substrate can dramatically influence the magnetic surface of cerium thin films in contrast to most of the uncorrelated thin films and strongly correlated transition metals. Our result implies that magnetic surface moments of $\alpha$ - $\mathrm{Ce}(111)$ can be suddenly disappeared by increasing lattice mismatch at the interface of a typical unreactive and non-diffusive substrate with cerium overlayers.
\end{abstract}

\section{INTRODUCTION}

Cerium even in its bulk form has been a long-standing challengeable system for theorists 1, 2] and experimentalists 3, 4] due to its demonstrated variable degrees of 4f states correlations, [5 7] resulting in various astonishing properties. [8, 9] Ce has been frequently appraised to present or criticize strongly correlated theories. 10 21] The reliability of the theoretical approaches has not yet been fully authenticated for this simple lanthanide metal. 22 Cerium displays an intriguing complex phase diagram containing a variety of solid states. 223] In this diagram there are two much more prominent phases, i.e., isostructural $\alpha$ and $\gamma$ solid states. A normal CurieWeiss law describes the magnetic susceptibility of the $\gamma$-phase, while the $\alpha$-phase shows an enhanced Pauli-like behavior. 8, 24] The $\gamma$-Ce is a ferromagnetic system with localized $4 \mathrm{f}$ states, whereas the $\alpha$-Ce is a paramagnetic system and its $4 \mathrm{f}$ states tend to be more hybridized with the valence bands. 25] The $\gamma$-Ce crystallizes in the fcc structure with $\sim 15 \%$ larger volume than its isostructural $\alpha$-Ce phase. The isostructural $\alpha \longleftrightarrow \gamma$ phase transition has been intensively studied. [13, 22, 25, 26] However, the physics underlying this unique transition is still under debate. 25] The difference between $\alpha$ - and $\gamma$-phases is attributed to dissimilarity in magnitude of the hybridization between $4 \mathrm{f}^{1}$ and $5 \mathrm{~d}^{1} 6 \mathrm{~s}^{2}$ states. 7] Now let us switch to its surface states, which can be even more problematic than the bulk states. The surface electronic states of cerium can be different from that of its bulk due to the appearance of surface states [27] and sensitivity of cerium to the symmetry of its surrounding environment. 14. Eriksson et al. [14], within their linearized muffin-tin orbitals (LMTO) calculations employing the Vosko-Wilk-

*Electronic address sjalali@phys.ui.ac.ir
Nusair local density approximation (VWN-LDA), found $\alpha$-Ce(100) surfaces to be $\gamma$-like, i.e., magnetically ordered. They 14] from their later result anticipated that the surface magnetism at the $\gamma$-Ce(100) layers should be more enhanced $\gamma$-like than the $\alpha$-Ce(100). The later anticipation might be a natural consequence of the Stoner's picture that, "the tendency toward magnetism should be increased near metal surfaces, because of the narrowing of the density of states that yields a Stoner enhancement in the susceptibility" 28]. Rothman and coauthors 32 experimentally by growing $\mathrm{Ce}(111)$ on a W(110) buffer confirmed the photo-emission spectra measurements of the $\mathrm{Gu}$ et al. 33. in which $\alpha$-like spectra were observed for a lattice parameter close to that of the $\gamma$-Ce. The later observations 32, 33] yield a clue to think about the possibility of vanishing magnetic moments at the surface of magnetic materials, the so-called dead layers [28]. The validity of the fact that the surface of $\alpha$-Ce(111) is $\gamma$-like has extensively been experimentally [27, 30] and theoretically [5, 31] verified. Nonetheless, there are unfortunately few reports on the $\gamma$-Ce thin films to justify about the magnetic tendency of the $\gamma$-Ce(111) surface to show whether it would be $\gamma$-like or $\alpha$-like. These motivated us to systematically calculate the surface magnetic moments of $\alpha$ - and $\gamma$-Ce(111) phases including spin and orbital polarizations as well as spin-orbit coupling which can be compulsory for cerium based systems. 34] We, in agreement with Ref. 14 and experiment 27, 30], show that the surface of nonmagnetic $\alpha$-phase tends to behave as a magnetic $\gamma$-like Ce compound. However, our result unexpectedly implies that the surface of $\gamma$-Ce(111) is $\alpha$-like. Indeed, our calculations demonstrate that the surface of magnetic $\gamma$-Ce is neither spin- nor orbital-polarized. Our result implies that an unreactive and non-diffusive substrate can cause a magnetic transition from magnetically ordered layers to a nonmagnetic layers at the surface of $\alpha$-Ce(111) thin films. We shall discuss how cerium can cause such an unexpected magnetic transition. 


\section{COMPUTATIONAL DETAILS}

This work has been carried out using the program package WIEN2k, 35] which allows to perform accurate all-electron full-potential augmented plane waves plus local orbital $(\mathrm{APW}+\mathrm{lo})$ [36, 37] band structure calculations of solids within the density functional theory (DFT). 38, 39] The Perdew-Burke-Ernzerhof generalized gradient approximation (PBE-GGA) 40] has been used for the exchange-correlation functional. The Ce Muffintin radii were set to $R_{M T}=1.8$ a.u. for the surface calculations. The expansion of the wave functions and charge densities were cut off by the $R_{M T} K_{\max }=7.0$ and $G_{\max }=12$ parameters, respectively. A set of $21 \times 21 \times 1$ $(19 \times 19 \times 19)$ special k-points has been used for integrations over the Brillouin zone of the supercell (unit cell) in the surface (bulk) calculations. The full relaxations were performed with the criterion of $1 \mathrm{mRy} / \mathrm{bohr}$ on the exerted forces. The relativistic effects were taken into account by including the spin-orbit ( $\mathrm{SO}$ ) coupling in a second variational procedure. Orbital polarizations were included to consider appropriate correlation in $4 \mathrm{f}$ Ce states using the LDA+U method. 41] Since LDA+U calculations can result in different solutions depending on initial conditions, care has been made to use an appropriate density matrix to ensure that the result has not been trapped in a local minimum.

\section{SUPERLATTICE SURFACE STRUCTURE}

Isostructural $\alpha$ - and $\gamma$-Ce crystallize in the Fm $\overline{3} \mathrm{~m}$ space group symmetry with the face-centered cubic (fcc) lattice structure, as shown in Fig. 1(a). We illustrate in Fig. 1 (b) a diagonal view of the fcc structure along the [111] direction normal to the (111) planes. The $\mathrm{z}$ axis is chosen to be perpendicular on the surfaces so that the $\mathrm{x}$ and y axes are parallel to them, as shown in Fig. 1 (b). Thin-films can be constructed by stacking cerium layers along our desired direction. In order to simulate the free $\mathrm{Ce}(111)$ surfaces, a symmetric supercell is created by adding vacuum spaces above and below Ce layers, as shown in Fig. 1 (c). To determine the vacuum thickness, total energies, work functions and exerted forces on the surface atoms were calculated versus various vacuum thicknesses. Our result shows that 25 bohr is sufficient for the vacuum thickness, as illustrated in Fig. 1 (c), to avoid interactions with the nearest neighbors of the slab along the Cartesian z axis.

\section{BULK PROPERTIES}

In order to more reliably simulate the surfaces of the $\alpha$ - and $\gamma-\mathrm{Ce}(111)$ so that the surface properties of the $\alpha$-phase can be distinguished from that of the $\gamma$-phase in consistent with experiment, we would first investigate whether the method used for the surface calcula-

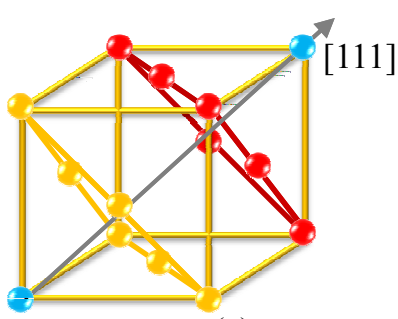

(a)

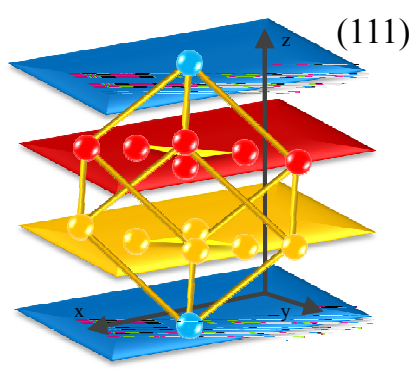

(b)

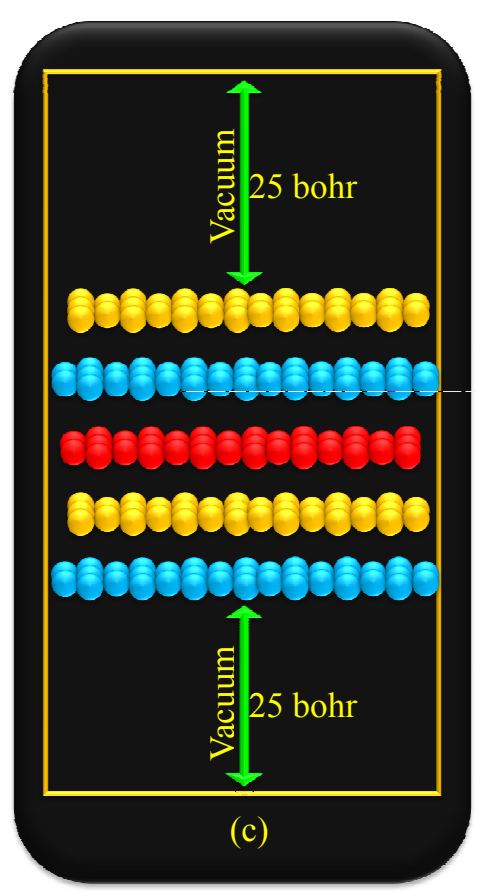

FIG. 1: "(color online)" Visualization of slab construction: (a) Fcc unit cell, where atoms on different planes normal to the [111] direction are distinguished, (b) Diagonal view of the fcc unit cell along the [111] direction indicating (111) surfaces. (c) Supercell containing 5 Ce(111) layers immersed symmetrically in a vacuum space as our simulated slab.

tions can properly reproduce the bulk properties of these two phases. The ground state equilibrium volumes were measured to be $28.17 \AA^{3}$ for the $\alpha$-Ce and $34.36 \AA^{3}$ for the $\gamma$-Ce. 42. The most apparent difference between these two phases is that the lattice parameter of the $\gamma$-phase is larger than that of the $\alpha$-phase. To authenticate whether the degree of $4 \mathrm{f}$ hybridization with other valence states can cause this change in lattice parameter, we have applied the LDA $+\mathrm{U}$ method 41] to the $\gamma$-phase and used the PBE-GGA [40] for the $\alpha$-phase. The spin-polarized PBEGGA including spin-orbit coupling (GGA+SP+SO) result shows an excellent agreement between our calculated lattice parameter, $4.808 \AA$, with experiment, $4.830 \AA$, 42. for the $\alpha$-phase. The later obtained excellent agreement using solely the GGA+SP+SO, with no further band correlated corrections, can be taken as an indication to the fact that the $4 \mathrm{f} \alpha$-Ce electrons tend to behave as bandlike itinerant electrons in agreement with Mott transition (MT) scenario. 11, 12] The GGA+SP+SO plus correlations ( $\mathrm{GGA}+\mathrm{SP}+\mathrm{SO}+\mathrm{U})$ among $4 \mathrm{f} \mathrm{Ce}$ electrons has been used with literature values of $6.1 \mathrm{eV}[15,43]$ and 4.4 $\mathrm{eV}$ [43] for the Hubbard U parameter in the $\gamma$-phase. We found that the former $\mathrm{U}$ value, $6.1 \mathrm{eV}$, results in a completely wrong lattice parameter for the $\gamma$-phase. For $\mathrm{U}=$ 0 , the minimum of the E-V curve of the localized $\gamma$-Ce coincides with the one of the $\alpha$-Ce. We could calculate the lattice parameter of the $\gamma$-phase using the later $\mathrm{U}$ value, 


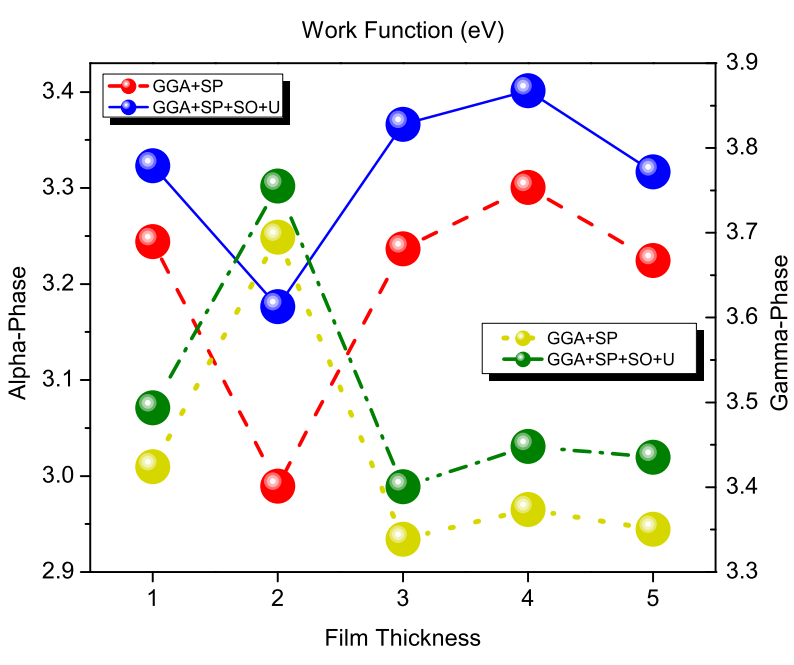

FIG. 2: "(color online)" Work function, $\phi(\mathrm{eV})$, versus number of cerium layers for $\alpha$-Ce(111), left vertical axis, and $\gamma$-Ce(111), right vertical axis, within GGA+SP and $\mathrm{GGA}+\mathrm{SP}+\mathrm{SO}+\mathrm{U}$.

which was corresponded [43] to the selected atomic configurations of $U=E\left(f^{3} S^{0}\right)+E\left(f^{1} S^{2}\right)-2 E\left(f^{2} S^{1}\right)=4.4 \mathrm{eV}$ for the promoted localized $4 \mathrm{f}$ electrons to more delocalized $6 \mathrm{~s}$ states. Our calculated lattice parameter of $\gamma$-Ce, $5.169 \AA$, using $\mathrm{U}=4.4 \mathrm{eV}$ is in excellent agreement with experiment, $5.160 \AA$, as well. [42] We obtained $11 \mathrm{kbar}$ for the $\alpha \leftrightarrow \gamma$-phase transition pressure which is comparable with experimental value of $8 \mathrm{kbar}$ and other theoretical results. [5, 13, 44, 45] Total magnetic moments were calculated to be 0.000 and $1.142 \mu_{B}$ for the $\alpha$ - and $\gamma$-phases, respectively, in agreement with the SIC-LSD results. 44]

\section{SURFACE PROPERTIES}

\section{A. Work Function}

Insofar as the accuracy of our surface calculations are concerned, our success in reproducing bulk properties may not seem to be sufficient. Thus, we would second offer an estimation concerning the accuracy of our surface calculations by reporting on the work function as an extremely surface sensitive quantity [46]. Therefore, we have also calculated the work function , $\phi(e V)$, as the minimum energy required to liberate an electron from the Fermi level $\left(E_{F}\right)$ to a point with negligible kinetic energy at the center of the vacuum of the slab. The work functions were obtained using the relation of $\phi=E_{v a c}-E_{F}$, where $E_{v a c}$ is estimated by the averaged electrostatic Coulomb potential at the midpoint of the vacuum of the slab and $E_{F}$ is the corresponding Fermi energy.

The work functions are given in Fig. 2 as a function
TABLE I: Spin magnetic moments inside the Muffin-tin sphere (MT), spin magnetic moments in the interstitial region (Int.), total spin magnetic moments per atom (Spin), orbital magnetic moment inside the Muffin-tin sphere (Orb.) and total magnetic moments per atom (Tot.) in $\mu_{B}$ versus number of layers (N) within the GGA+SP+SO for the $\alpha$-Ce(111) thin films. We calculated total spin moment as Spin = MT+Int., for $\mathrm{N}=1$ and as Spin $=\mathrm{MT}+\frac{1}{2}$ Int., for $\mathrm{N} \geq 2$. Total magnetic moment is calculated to be Tot. = Spin + Orb., for every N.

\begin{tabular}{lccccc}
\hline \hline $\mathrm{N}$ & 1 & 2 & 3 & 4 & 5 \\
\hline $\mathrm{MT}$ & 0.61 & 1.02 & 0.53 & 0.49 & 0.65 \\
Int. & 0.37 & 1.54 & 0.85 & 0.71 & 0.75 \\
Spin & 0.98 & 1.79 & 0.96 & 0.85 & 1.03 \\
Orb. & -0.21 & -0.45 & -0.22 & -0.22 & -0.39 \\
Tot. & 0.77 & 1.34 & 0.74 & 0.63 & 0.64 \\
\hline \hline
\end{tabular}

of the film thickness for both of the $\alpha$ - and $\gamma$-C(111) clean surfaces. As shown in Fig. 2 , the $\mathrm{SO}+\mathrm{U}$ causes to reduce the work function for both of the phases. The result shows that the work functions of the $\gamma$-phase is well converged through our five thin layers. The convergence of the $\gamma$-phase, as shown in Fig. 2 is more reliable than that of the $\alpha$-phase. Fluctuation of the $\alpha$-phase between fourth and fifth layers shows also a small change of about $2 \%$ in the work functions. The result, Fig. 2, indicates that the fluctuation of the work function is not substantially affected by the spin-orbit coupling and/or $\mathrm{LDA}+\mathrm{U}$ correlations. The result shows that the work function oscillate as a function of the number of layers with the period of $\lambda=2$ layers. The oscillation indicates how the quantum size effects (QSEs) 47] can affect the results. The period of oscillation, as shown in Fig. 2, is less than the period of the "abcabc" pattern of the fcc stacking. This can be taken as an indication to the fact that here oscillations of the work function originates more dominantly from the quantum size effects (QSEs) at nanoscale than the period of thin films stacking due to the symmetry of the structure 47]. Our result shows that the work functions of the $\gamma$-phase are larger than those of the $\alpha$-phase, for every number of layers. The later result is in agreement with the LMTO result, 14 where the work functions were calculated to be $3.5 \mathrm{eV}$ for $\alpha$ - $\mathrm{Ce}(100)$ and $4.2 \mathrm{eV}$ for $\gamma-\mathrm{Ce}(100)$. Our calculated work functions are closer to the experimental value, $2.9 \pm 0.2$, [48] than the LMTO values. These results, in accord with the MT[11, 12] and Kondo volume collapse (KVC) 13] pictures, which reconfirm that the $4 \mathrm{f}$ electrons tend to be more localized in the $\gamma$-Ce than that of $\alpha$-Ce, may ensure that our subsequent surface results might be reliable as well. 


\section{B. Magnetic Moment}

Turning to the goal of this paper, we first report on the magnetic moments for the $\alpha-\mathrm{Ce}(111)$ thin films and then for the $\gamma-\mathrm{Ce}(111)$ thin films. The magnetic moments are listed in Tab. \for the $\alpha-\mathrm{Ce}(111)$ thin films. The result shows that the direction of the total spin moments is opposite to that of the orbital moments. However, total magnetic moments are still considerable for the surface of $\alpha$-Ce. This implies that the surface of the nonmagnetic $\alpha$-Ce is $\gamma$-like in agreement with experiments [27, 30] and other theoretical results [5, 31] as well as the pioneer work of Eriksson and coworkers [14]. The result, as presented in Tab. I) shows that the total magnetic moment changes by only a tiny percentage of about $1 \%$ on going from fourth to the fifth layer. Here in fact, cancellation errors between spin and orbital contributions cause such a tiny percentage in the total magnetic moments. The surface of $\alpha-\mathrm{Ce}(111)$ is well known to be $\gamma$-like experimentally [27, 30] and theoretically [5, 31]. Therefore, the result presented in Tab. I can qualitatively corroborate the idea that the surface of $\alpha$ - $\mathrm{Ce}(111)$ is magnetically ordered in agreement with theory [5, 31] and experiment [27, 30]. For the $\gamma$-Ce(111) thin films, we did not present similar table, because our result shows that such a table contains nothing more than zero moments. The accuracy to which we have calculated the magnetic moments is $\pm 0.01 \mu_{B}$ for the $\gamma$-Ce(111). Thereby if we present a table for the magnetic surfaces of $\gamma-\mathrm{Ce}(111)$, the table contains ignorable values of $0.00 \pm 0.01 \mu_{B}$. Such ignorable values are obtained for all components of the total magnetic moments, i.e. according to the abbreviations given and defined in the caption of Tab. I. MT, Int., Spin, Orb. and as a result Tot., from $\mathrm{N}=1$ to $\mathrm{N}=5$. Therefore in essence, inversely the surface of the magnetic $\gamma$-Ce is found within our ab initio DFT calculations to be $\alpha$-like. Thus, we predict that the free surfaces of the $\gamma$ phase constitute dead layers at zero temperature. Such a contradictory situation in these isostructural phases concerning different magnetic behaviors of their bulks when compared with their free (111) surfaces can be considered as another physical property for cerium. Our result confirms the experimental works preformed by Rothman and coauthors 32] and $\mathrm{Gu}$ et al. 33] where $\alpha$-like spectra were measured for a lattice parameter close to that of the $\gamma$-Ce. This represents another success of the density functional theory (DFT) in fundamentally predicting complicated 4f-electron nanosystems from first principles of quantum mechanics without assuming any experimental data. It is to some extent hard to expect that there can be found another case in nature similar to cerium with two isostructural phases and opposite magnetic surfaces compared to their corresponded bulk counterparts. This implies that cerium due to its demonstrated capacity in reproducing two entirely opposite magnetic surface states might be considered as a unique case. Thereby, Ce once more serves as an interesting case to appraise the validity of our fundamental understanding based on the density functional theory (DFT).

\section{EFFECT OF LATTICE MISMATCH}

From our hypothetical freestanding surface study one may also deduce that a lattice mismatch at the interface with a typical unreactive and non-diffusive substrate can play an important role on the magnitude of the magnetic moments in Ce thin films. The sensitivity of cerium thin films to the effects of lattice mismatch can be inferred by the fact that the differences between $\alpha$ - and $\gamma$-phases may be resulted from the larger lattice parameter of the $\gamma$-Ce than that of the $\alpha$-Ce. The unreactive and non-diffusive substrate affects the lattice mismatch of the cerium overlayers. The lattice mismatch at the interface of a typical $\mathrm{Ce}(111) /$ substrate causes to change lattice parameter of the cerium thin films. The change of lattice parameter in Ce can give rise to transition from $\alpha$ - to $\gamma$-phase. In uncorrelated thin films the change of lattice mismatch to some extent may not drastically change the electronic structures. This could be also the case for strongly correlated materials. For instance, the effects of gold and cooper substrates on the ferromagnetic transition metals were refuted in leading to magnetically dead layers. [49] Lattice mismatch on cerium is important not only due to its 4f-electrons, but also more importantly due to existing isostructural $\alpha$ - and $\gamma$-phases in the vicinity of each other. This point might be of significant importance in nanotechnology, as in this case one can control the surface magnetic moments ranging from enhanced magnetically ordered surfaces to magnetically dead layers by changing unreactive and non-diffusive substrates. Therefore, we predict that more lattice mismatch in $\alpha$-Ce thin films can give rise to less magnetic surface. The magnetic surface of $\alpha$-Ce thin films can suddenly disappear by increasing lattice mismatch towards lattice parameter of $\gamma$-Ce. The later point shows that how an unreactive and nondiffusive substrate can significantly influence the cerium thin films.

\section{DENSITY OF STATES (DOS)}

To realize the underlying physics of the above discussed property, we give in Figs. 3 the density of states (DOS) curves. As shown in Fig. 3 (a), the up and down bulk DOSs are symmetric with respect to each other for the $\alpha$-Ce, which is not the case for the $\gamma$-Ce, as shown in Fig. 3 (f). Therefore, one anticipates to find (zero) nonzero magnetic moment for the ( $\alpha$-phase) $\gamma$-phase. As can be seen from Figs. 3 (a) and (f), including correlations among 4 f-electrons cannot affect the later anticipation. The calculated surface DOS curves within LDA are shown in Figs. 3 (b) and (g) for the $\alpha$ - and $\gamma$-Ce(111), respectively. One notices from the later surface DOSs that the $\alpha$ - and $\gamma$-phases would exchange their magnetic behaviors by going from their bulks to their surfaces. As 


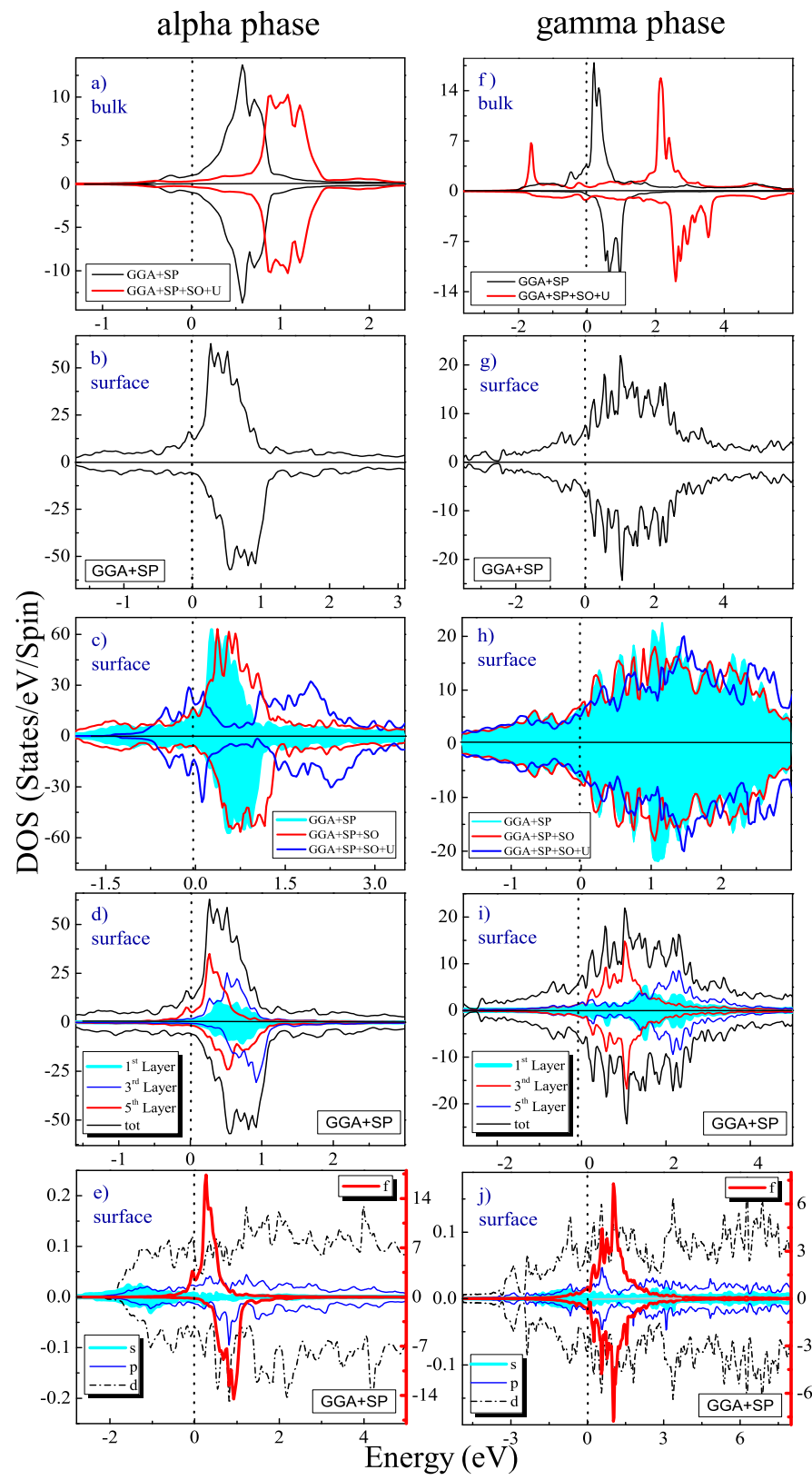

FIG. 3: "(color online)" Bulk and free surface DOSs of the $\alpha$-Ce (left panel) and $\gamma$-Ce (right panel).

shown in Fig. 3 (g), the up and down bulk DOSs are symmetric with respect to each other for the $\gamma$-Ce(111) surface, which is not the case for the $\alpha$-Ce(111), as shown in Fig. 3 (b). Therefore, in contrast to what has been already anticipated above for the bulk counterparts, at the surface the $\alpha$-Ce(111) is magnetic whereas $\gamma$-Ce(111) is nonmagnetic. This shows that the above mentioned anticipation can be affected by the surface states. This means that the surface of the $\alpha$-Ce is $\gamma$ - like whereas the surface of the $\gamma$-Ce is $\alpha$-like. As can be seen from Figs. 3 (c) and (h), including spin-orbit (SO) coupling and correlations among 4 f-electrons cannot change back the reversed surface magnetic properties to that of the bulks. To shed light into the electronic structures of interior layers, DOSs at various layers of our slab containing totaly 5 symmetric layers were also calculated within the $\mathrm{GGA}+\mathrm{SP}+\mathrm{SO}+\mathrm{U}$ and the results are given in Figs. 3 (d) and (i) for the $\alpha$ - and $\gamma$-Ce(111), respectively. The result shows that all the layers, i.e., deeper and/or shallower layers, are magnetic for the $\alpha-\mathrm{Ce}(111)$ and nonmagnetic for the $\gamma$-Ce(111). The calculated Partial DOSs employing $\mathrm{GGA}+\mathrm{SP}+\mathrm{SO}+\mathrm{U}$ are shown in Figs. 3 (e) and $(\mathrm{j})$ for the $\alpha$ - and $\gamma-\mathrm{Ce}(111)$, respectively. The result, as can be seen from Fig. 3 (e), shows that the surface magnetic properties of the nonmagnetic $\alpha$-phase is due to the partial 4f-states. The result, as shown in Fig. 3 (j), also clarify the role of $4 \mathrm{f}$-states in suppressing the magnetic moments at the surface of magnetic $\gamma$-phase.

\section{CONCLUSION}

In conclusion, we have presented an evidence in favor of entirely different magnetic orderings at the $\alpha-$ and $\gamma-\mathrm{Ce}(111)$ clean surfaces as opposed to their bulk counterparts. Bulk magnetic moments were calculated to be zero and $1.14 \mu_{B}$ for isostructural enhanced Paulilike $\alpha$-Ce and Curie-Weiss $\gamma-$ Ce phases, respectively, in agreement with other theoretical calculations. Inversely, surface magnetic moments were calculated to be nonzero for the $\alpha-\mathrm{Ce}(111)$ and zero for the $\gamma-\mathrm{Ce}(111)$ thin film layers. The contradictory situation that arises from different magnetic transitions in these isostructural phases from their bulks to their free (111) surfaces can be considered as another unexpected property for cerium and attributed to different $4 \mathrm{f}$ hybridizations with the other valence states at diverse surface circumstances. Our result predicts that the nonmagnetic $\alpha$-phase tends to produce magnetically ordered $\gamma$-like surface layers, whereas the magnetic $\gamma$-phase has a tendency to form $\alpha$-like dead layers. In summery, we have shown that the surface of $\alpha$-Ce is $\gamma$-like, while the surface of $\gamma$-Ce is $\alpha$-like. Within the later result one concludes that how can cerium be of significant importance in nanotechnology, because physical properties of cerium thin films can be drastically influenced by those of unreactive and non-diffusive substrates which can impose large lattice mismatch. Our result implies that the significant effects of unreactive and non-diffusive substrates on the adatoms can be expected solely from cerium due to its 4 f-electrons and isostructural $\alpha$ - and $\gamma$-phases as well as the discussed entirely different magnetic ordering of the isostructural phases at their corresponded surfaces.

\section{Acknowledgments}

This work is supported by University of Isfahan (UI), Isfahan, Iran. S.J.A. is thankful to Computational Nanotechnology Supercomputing Center Institute for Re- 
[1] O. Eriksson, J.M. Wills, and A.M. Boring, Phys. Rev. B 46, 12981-12989 (1992).

[2] Y. Wang et al., Phys. Rev. B 78, 104113 (2008).

[3] Hitoshi Homma, Kai-Y. Yang, and Ivan K. Schuller, Phys. Rev. B 36, 9435-9438 (1987).

[4] Yu. S. Dedkov et al., Phys. Rev. B 76, 073104 (2007).

[5] D.L. Price, Phys. Rev. B 60, 10588 (1999).

[6] B.E. Light et al., Phys. Rev. B 69, 024419 (2004).

[7] B. Amadon, F. Jollet, and M. Torrent, Phys. Rev. B 77, 155104 (2008).

[8] J.S. Olsen et al., Physica B \& C 133, 129 (1985).

[9] J.W. van der Eb, A.B. Kuźmenko, and D. van der Marel, Phys. Rev. Lett. 86, 3407 (2001).

[10] L. Pauling, J. Am. Chem. Soc. 69, 542 (1947).

[11] N.F. Mott, Rev. Mod. Phys. 40, 677 (1968); MetalInsulator Transitions (Taylor \& Francis, London, 1990).

[12] B. Johansson, Philos. Mag. 30, 469 (1974).

[13] J.W. Allen and R.M. Martin, Phys. Rev. Lett. 49, 1106 (1982); M. Lavagna, C. Lacroix, and M. Cyrot, Phys. Lett. 90A, 210 (1982); J.W. Allen and L.Z. Liu, Phys. Rev. B 46, 5047 (1992).

[14] O. Eriksson et al., Phys. Rev. B 43, 3137-3142 (1991).

[15] V.I. Anisimov and O. Gunnarsson, Phys. Rev. B 43, 7570 (1991).

[16] V.I. Anisimov et al., J. Phys.: Condens. Matter 9, 7359 (1997).

[17] A.I. Lichtenstein and M.I. Katsnelson, Phys. Rev. B 57, 6884 (1998).

[18] G. Eliashberg and H. Capellmann, Piśma Zh. Eksp. Teor. Fiz 67, 111 (1998); JETP Lett. 67, 125 (1998).

[19] A.V. Nikolaev and K.H. Michel, Eur. Phys. J. B 9, 619 (1999); 17, 12 (2000); Phys. Rev. B 66, 054103 (2002).

[20] Kristjan Haule et al., Phys. Rev. Let. 94, 036401 (2005).

[21] G. Kotliar et al., Rev. Mod. Phys. 78, 865 (2006).

[22] A.K. McMahan, K. Held, and R.T. Scalettar, Phys. Rev. B 67, 075108 (2003).

[23] R. Ramirez, and L.M. Falicov, Phys. Rev. B 3, 2425 (1971).

[24] A.P. Murani et al., Phys. Rev. B 48, 13981-13984 (1993).

[25] B. Amadon et al., Phys. Rev. Lett. 96, 066402 (2006).

[26] J. Lægsgaard and A. Svane, Phys. Rev. B 59, 3450-3459
(1999).

[27] E. Weschke et al., Phys. Rev. B 58, 3682 (1998).

[28] N. Stojić, J.W. Davenport, M. Komelj, and J. Glimm, Phys. Rev. B 68, 094407 (2003).

[29] Y. Baer et al., Phys. Rev. B 44, 9108 (1991).

[30] E. Weschke et al., Phys. Rev. B 44, 8304 (1991).

[31] O. Eriksson et al., Surface Science 382, 93 (1997).

[32] J. Rothman et al., Jou. of Mag. and Mag. Mat., 198, 276 (1999).

[33] C. Gu, et al., Phys. Rev. lett. 67, 1622 (1991).

[34] S. Jalali Asadabadi, Phys. Rev. B 75, 205130 (2007).

[35] P. Blaha et al., WIEN2K, "An Augmented Plane Waves + Local Orbitals Program for Calculating Crystal Properties," Karlheinz Schwarz, Techn. Universitat Wien, Austria, ISBN 3-9501031-1-2 (2001).

[36] E. Sjöstedt, L. Nordström, and D. J. Singh, Solid State Commun. 114, 15 (2000).

[37] G.K.H. Madsen et al., Phys. Rev. B 64, 195134 (2001).

[38] P. Hohenberg and W. Kohn, Phys. Rev. 136, 864 (1964).

[39] W. Kohn and L. J. Sham, Phys. Rev. 140, A1133 (1965).

[40] J.P. Perdew, K. Burke, and M. Ernzerhof, Phys. Rev. Lett. 77, 3865 (1996).

[41] V.I. Anisimov et al., Phys. Rev. B 48, 16929 (1993); M. T. Czyżyk and G. A. Sawatzky, Phys. Rev. B 49, 14211 (1994); E. Sjöstedt, L. Nordström, and D. J. Singh, Solid State Commun. 114, 15 (2000).

[42] J. Donohu, The Stucture of the Elements (John Wiley \& Sons, Inc., New York, 1974).

[43] M. Cococcioni and S. de Gironcoli, Phys. Rev. B 71, 035105 (2005).

[44] A. Svane, Phys. Rev. B 53, 4275 (1996).

[45] B. Johansson et al., Phys. Rev. Lett. 74, 2335 (1995).

[46] Y. Baer, M. Grioni, and D. Malterre, Phys. Rev. B 44, 9108-9109 (1991).

[47] M. Rafiee, and S. Jalali Asadabadi, Comp. Mat. Scie. 47, 584-592 (2009).

[48] D.E. Eastman, Phys. Rev. B 2, 1 (1970).

[49] L. Liebermann, J. Clinton, D.M. Edwards and J. Mathon, Phys. Rev. B 25, 232 (1970). 